Vietnam Journal of Mechanics, VAST, Vol.34, No.1 (2012), pp. $7-17$

\title{
ON A CLASS OF NON-LINEAR DIFFERENTIAL EQUATIONS WITH EXACT SOLUTIONS
}

\author{
Dao Huy Bich ${ }^{1}$, Nguyen Dang Bich ${ }^{2}$ \\ ${ }^{1}$ Hanoi University of Science, $V N U$ \\ ${ }^{2}$ Institute for Building Science and Technology
}

\begin{abstract}
The present paper deals with a class of non-linear ordinary second-order differential equations with exact solutions. A procedure for finding the general exact solution based on a known particular one is derived. For illustration solutions of some non-linear equations occured in many problems of solid mechanics are considered.

Key words: Non-linear differential equation, general exact solution, varying coefficient iteration method, procedure for finding exact solution.
\end{abstract}

\section{INTRODUCTION}

Generally for seeking an exact solution of a non-linear differential equation it is necessary to find an appropriate transformation deriving the non-linear equation to a linear one, but finding of such transformation is very complicated.

In fact many problems of solid mechanics reduce to different types of non-linear differential equations, solutions of which can demonstrate specific effects, however through only exact solutions these effects can be observed profoundly. Hence finding exact solutions becomes very important in researching non-linear mechanical problems.

The present paper introduces an idea and a procedure to find general exact solutions of a class of non-linear ordinary second-order differential equations based on known particular solutions. If a particular exact solution is known, then a general exact solution can be found, but for a received approximate particular one, a general solution may be obtained approximately with desired accuracy by the varying coefficient iteration method.

\section{IDEA AND PROCEDURE FOR FINDING EXACT SOLUTION}

Consider a non-linear second-order differential equation

$$
\begin{aligned}
& \frac{d}{d t}\left(a_{1} \frac{\dot{x}}{x+d}+a_{2} x+a_{3}+\frac{b}{x+d}\right)+\left(a_{1} \frac{\dot{x}}{x+d}+a_{2} x+a_{3}+\frac{b}{x+d}\right)^{2}+ \\
& +\frac{d}{d t}\left(b_{1} \frac{\dot{x}}{x+d}+b_{2} x+b_{3}+\frac{b}{x+d}\right)-\left(b_{1} \frac{\dot{x}}{x+d}+b_{2} x+b_{3}+\frac{b}{x+d}\right)^{2}=0 .
\end{aligned}
$$

where $a_{i}, b_{i}(i=1,2,3), b, d$ are constants, $x(t)$ is unknown function of $t$. 
Using notation

$$
\begin{aligned}
& \frac{\dot{u}}{u}=a_{1} \frac{\dot{x}}{x+d}+a_{2} x+a_{3}+\frac{b}{x+d}, \\
& \frac{1}{2} \frac{\dot{v}}{v}=b_{1} \frac{\dot{x}}{x+d}+b_{2} x+b_{3}+\frac{b}{x+d},
\end{aligned}
$$

equation (1) can be rewritten as follows:

$$
\frac{d}{d t}\left(\frac{\dot{u}}{u}\right)+\left(\frac{\dot{u}}{u}\right)^{2}+\frac{d}{d t}\left(\frac{1}{2} \frac{\dot{v}}{v}\right)-\left(\frac{1}{2} \frac{\dot{v}}{v}\right)^{2}=0
$$

or in the other form

$$
\frac{d}{d t}\left(\frac{\dot{u}}{u}+\frac{1}{2} \frac{\dot{v}}{v}\right)+\left(\frac{\dot{u}}{u}-\frac{1}{2} \frac{\dot{v}}{v}\right)\left(\frac{\dot{u}}{u}+\frac{1}{2} \frac{\dot{v}}{v}\right)=0 .
$$

Eq. (1) has a particular solution when

$$
\frac{\dot{u}}{u}+\frac{1}{2} \frac{\dot{v}}{v}=0
$$

or

$$
\left(a_{1}+b_{1}\right) \frac{\dot{x}}{x+d}+\left(a_{2}+b_{2}\right) x+a_{3}+b_{3}+\frac{2 b}{x+d}=0 .
$$

The particular solution can be obtained from (7) as:

(i). A function of $t$

$$
x+d=-\frac{a_{3}-a_{2} d+b_{3}-b_{2} d}{2\left(a_{2}+b_{2}\right)}+\frac{\sqrt{\delta}}{2\left(a_{2}+b_{2}\right)} t h\left[\frac{\sqrt{\delta} t}{2\left(a_{1}+b_{1}\right)}+\varphi\right],
$$

where $\delta=\left(a_{3}-a_{2} d+b_{3}-b_{2} d\right)^{2}-8 b\left(a_{2}+b_{2}\right), \varphi$ - integral constant.

(ii). A constant

$$
\begin{aligned}
& x+d=-\frac{a_{3}-a_{2} d+b_{3}-b_{2} d}{2\left(a_{2}+b_{2}\right)}+\frac{\sqrt{\delta}}{2\left(a_{2}+b_{2}\right)}, \\
& x+d=-\frac{a_{3}-a_{2} d+b_{3}-b_{2} d}{2\left(a_{2}+b_{2}\right)}-\frac{\sqrt{\delta}}{2\left(a_{2}+b_{2}\right)} .
\end{aligned}
$$

In order to represent funtion $u$ through funtion $v$ based on Eq. (5) it can be obtained

$$
\frac{\dot{u}}{u}=-\frac{1}{2} \frac{\dot{v}}{v}+\frac{C_{2} v}{C_{1}+C_{2} \int_{0}^{t} v d t}
$$

thus

$$
u=\frac{1}{v^{1 / 2}}\left(C_{1}+C_{2} \int_{0}^{t} v d t\right)
$$

where $C_{1}, C_{2}$ - integral constants. 
Subtracting side by side of Eqs.(2) and (3) and taking (11) into account yields

$$
\left(a_{1}-b_{1}\right) \frac{\dot{x}}{x+d}+\left(a_{2}-b_{2}\right)(x+d)+a_{3}-a_{2} d-\left(b_{3}-b_{2} d\right)=\frac{C_{2} v}{C_{1}+C_{2} \int_{0}^{t} v d t}-\frac{\dot{v}}{v} .
$$

Eq. (13) can lead to a linear differential equation with respect to unknown $\frac{1}{x+d}$

$$
\frac{d}{d t}\left(\frac{1}{x+d}\right)+\frac{1}{a_{1}-b_{1}}\left[\frac{C_{2} v}{C_{1}+C_{2} \int_{0}^{t} v d t}-\frac{\dot{v}}{v}-\left(a_{3}-a_{2} d\right)+\left(b_{3}-b_{2} d\right)\right] \frac{1}{x+d}=\frac{a_{2}-b_{2}}{a_{1}-b_{1}}
$$

Eq. (14) is derived from Eq.(1) by use of an equivalent transformation, consequently it is equivalent with Eq.(1), Eq.(14) is a linear differential equation with varying coefficient, that is in this case

$$
\frac{1}{a_{1}-b_{1}}\left[\frac{C_{2} v}{C_{1}+C_{2} \int_{0}^{t} v d t}-\frac{\dot{v}}{v}-\left(a_{3}-a_{2} d\right)+\left(b_{3}-b_{2} d\right)\right]
$$

Following the idea of varying coefficient iteration method [2], at the first iteration this coefficient can be evaluated by particular solutions (8), (9) and (10).

Denote the particular solution of Eq.(1) by $x_{1}$, then Eq. (3) and Eq. (7) give:

$$
\begin{gathered}
2 b_{1} \frac{\dot{x}_{1}}{x_{1}+d}+2 b_{2} x_{1}+2 b_{3}+\frac{2 b}{x_{1}+d}=\frac{\dot{v}}{v} \\
\left(a_{1}+b_{1}\right) \frac{\dot{x}_{1}}{x_{1}+d}+\left(a_{2}+b_{2}\right) x_{1}+\left(a_{3}+b_{3}\right)+\frac{2 b}{x_{1}+d}=0 .
\end{gathered}
$$

Subtracting side by side of two these equations yields:

$$
\left(a_{1}-b_{1}\right) \frac{\dot{x}_{1}}{x_{1}+d}+\left(a_{2}-b_{2}\right) x_{1}+a_{3}-b_{3}=-\frac{\dot{v}}{v},
$$

from that

$$
v=\left(x_{1}+d\right)^{-\left(a_{1}-b_{1}\right)} \exp \left[-\left(a_{2}-b_{2}\right) \int_{0}^{t}\left(x_{1}+d\right) d t\right] \exp \left[\left(-a_{3}+a_{2} d+b_{3}-b_{2} d\right) t\right] .
$$

Consequently at first iteration Eq. (14) has a general solution as follows

$$
\frac{1}{x+d}=\frac{1}{\mu}\left[\frac{a_{2}-b_{2}}{a_{1}-b_{1}} \int_{0}^{t} \mu d t+C\right]
$$


where $C$ is an integral constant and

$$
\mu=\left(x_{1}+d\right) \exp \left[\frac{a_{2}-b_{2}}{a_{1}-b_{1}} \int_{0}^{t}\left(x_{1}+d\right) d t\right]\left(C_{1}+C_{2} \int_{0}^{t} v d t\right)^{\frac{1}{a_{1}-b_{1}}} .
$$

In order to verify the general solution (19), (20) satisfying exactly or not Eq.(14), i.e. the equivalent equation of Eq.(1), a procedure is established as following. Taking logarithm both sides of Eq.(19) and differentiating with respect to $t$ yield

$$
\frac{\frac{d}{d t}\left(\frac{1}{x+d}\right)}{\frac{1}{x+d}}=-\frac{\dot{\mu}}{\mu}+\frac{\frac{a_{2}-b_{2}}{a_{1}-b_{1}} \mu}{\frac{a_{2}-b_{2}}{a_{1}-b_{1}} \int_{0}^{t} \mu d t+C}=-\frac{\dot{\mu}}{\mu}+\frac{a_{2}-b_{2}}{a_{1}-b_{1}}(x+d)
$$

In other side Eq.(14) reduces to

$$
\begin{gathered}
\frac{\frac{d}{d t}\left(\frac{1}{x+d}\right)}{\frac{1}{x+d}}+\frac{1}{a_{1}-b_{1}}\left[\frac{C_{2} v}{C_{1}+C_{2} \int_{0}^{t} v d t}-\frac{\dot{v}}{v}-\left(a_{3}-a_{2} d\right)+\left(b_{3}-b_{2} d\right)\right]=\frac{a_{2}-b_{2}}{a_{1}-b_{1}}(x+d) . \\
\text { Eliminating } \frac{\frac{d}{d t}\left(\frac{1}{x+d}\right)}{\frac{1}{x+d}} \text { from obtained equations results } \\
\frac{1}{a_{1}-b_{1}}\left[\frac{C_{2} v}{C_{1}+C_{2} \int_{0}^{t} v d t}-\frac{\dot{v}}{v}-\left(a_{3}-a_{2} d\right)+\left(b_{3}-b_{2} d\right)\right]=\frac{\dot{\mu}}{\mu},
\end{gathered}
$$

and from (20) it follows:

$$
\frac{\dot{\mu}}{\mu}=\frac{\dot{x}_{1}}{x_{1}+d}+\frac{a_{2}-b_{2}}{a_{1}-b_{1}}(x+d)+\frac{C_{2} v}{C_{1}+C_{2} \int_{0}^{t} v d t} \frac{1}{a_{1}-b_{1}} .
$$

Substitution of (22) into (21) reduces to Eq. (17)

$$
\left(a_{1}-b_{1}\right) \frac{\dot{x}_{1}}{x_{1}+d}+\left(a_{2}-b_{2}\right)\left(x_{1}+d\right)+a_{3}-a_{2} d-\left(b_{3}-b_{2} d\right)=-\frac{\dot{v}}{v} .
$$

Summing up side by side of this equation and equation (15) we can get again Eq.(16). Indeed Eq.(16) is the equation for seeking particular solution to Eq.(1).

In other side we can verify directly that, the general solution (19), (20) satisfies exactly the original equation (1).

Remark: As seen that Eq.(14) is equivalent to Eq.(1), it has general exact solution (19), (20) when $x_{1}$ is a particular solution of Eq.(1). So that at first iteration if $x_{1}$ is 
a particular exact solution, then the expression (19), (20) is a general exact solution of Eq.(14). If $x_{1}$ is a particular approximate solution, then (19), (20) is a general approximate solution of Eq. (14) at first iteration. For obtaining a general approximate solution with desired accuracy it need to fulfill successive iteration by the varying coefficient method. In the present paper we consider only the case when $x_{1}$ is a particular exact solution.

Consequently, the idea of the proposed method is to derive a non-linear differential equation to a linear differential equation with varying coefficient. This varying coefficient is determined by a particular solution of the original equation. If the particular solution is exact, the general exact solution can be obtained, if the particular solution is approximate then the approximated general solution can be evaluated with desired accuracy.

Procedure can be established by following steps:

Step 1: Seeking a particular solution of linear equation (16).

Step 2: Calculate $v$ according to (18).

Step 3: Calculate $\mu$ according to (20).

Step 4: Seeking a general solution according to (19).

The solution of Eq.(14), i.e. the expressions (19), (20) consists of four integral constants and particular solution, which are chosen appropriately for each concrete problem.

\section{PARTICULAR CASES OF EQ. (1)}

3.1. Case 1: $b_{1}=0, a_{2}=b_{2}=0, b=0, d=0$

Eq. (1) is of the form:

$$
\ddot{x}+2 a_{3} \dot{x}+\frac{a_{3}^{2}-b_{3}^{2}}{a_{1}} x=\left(1-a_{1}\right) \frac{\dot{x}^{2}}{x} .
$$

From Eq. (16) the particular solution is found directly

$$
x_{1}=\exp \left[-\frac{a_{3}+b_{3}}{a_{1}} t\right]
$$

the integral constant is taken $\varphi=0$. According to Eq.(18) function $v$ is calculated as $v=e^{2 b_{3} t}$ and substituting the obtained value $v$ into (20) yields

$$
\mu=\left[e^{-\left(a_{3}+b_{3}\right) t}\left(C_{1}+\frac{C_{2}}{2 b_{3}} e^{2 b_{3} t}\right)\right]^{1 / a_{1}},
$$

where $C_{1}, C_{2}$ are integral constants. The expression $\mu$ can be rewritten in other form

$$
\mu=\left[A e^{-a_{3} t} \operatorname{ch}\left(b_{3} t+\gamma\right)\right]^{\frac{1}{a_{1}}}, \quad A, \gamma-\text { integral constants. }
$$

By use of Eq. (19), the general solution of Eq. (23) is found

$$
x=B\left[e^{-a_{3} t} \operatorname{ch}\left(b_{3} t+\gamma\right)\right]^{\frac{1}{a_{1}}},
$$

where $B, \gamma$ are integral constants. 
If the coefficient of the Eq. (23) are denoted by

$$
a_{3}=\nu, \frac{a_{3}^{2}-b_{3}^{2}}{a_{1}}=\omega^{2}, \text { i.e. } b_{3}=\sqrt{\nu^{2}-a_{1} \omega^{2}},
$$

then with $\nu^{2}-a_{1} \omega^{2}<0$, the solution has form

$$
x=B\left[e^{-\nu t} \cos \left(t \sqrt{a_{1} \omega^{2}-\nu^{2}}+\gamma\right)\right]^{\frac{1}{a_{1}}} .
$$

The general solution (25) for Eq. (23) was found in [3] by other method.

3.2. Case 2: $a_{1}-b_{1}=-1, a_{2}=b_{2}, b=0, d=0$

Eq. (1) in this case has the form:

$$
\ddot{x}-2 \frac{\dot{x}^{2}}{x}+\frac{2\left(a_{1} a_{3}-b_{1} b_{3}\right)}{a_{1}+b_{1}} \dot{x}+\frac{2 a_{2}\left(a_{3}-b_{3}\right) x^{2}}{a_{1}+b_{1}}+\frac{\left(a_{3}^{2}-b_{3}^{2}\right) x}{a_{1}+b_{1}}=0 .
$$

According to Eqs. (8), (10) two particular solutions are obtained

$$
\begin{gathered}
x_{11}=-\frac{a_{3}+b_{3}}{4 a_{2}}+\frac{a_{3}+b_{3}}{4 a_{2}} t h\left[\frac{a_{3}+b_{3}}{2\left(a_{1}+b_{1}\right)} t+\varphi\right], \\
x_{12}=-\frac{a_{3}+b_{3}}{2 a_{2}} .
\end{gathered}
$$

Based on Eqs.(14), (19) and (20) the general solution of Eq.(26) has the form:

$$
\frac{1}{x}=\frac{C\left(C_{1}+C_{2} \int_{0}^{t} v d t\right)}{x_{1}}
$$

In the formulas (29) figure four integral constants $C, C_{1}, C_{2}, \varphi$ and the particular solution $x_{1}$, which need to be chosen appropriately. Such choice can be established as follows

$$
\frac{1}{x}=\frac{C C_{1}}{x_{11}}+\frac{C C_{2} \int_{0}^{t} v d t}{x_{12}} .
$$

Carrying out some simple calculations in Eq.(27) yields

$$
\frac{1}{x_{11}}=-\frac{2 a_{2}}{a_{3}+b_{3}}\left(1+e^{2 \varphi} e^{\frac{a_{3}+b_{3}}{a_{1}+b_{1}} t}\right)=-\frac{2 a_{2}}{a_{3}+b_{3}}+A e^{\frac{a_{3}+b_{3}}{a_{1}+b_{1}} t}
$$

where $A=-\frac{2 a_{2}}{a_{3}+b_{3}} e^{2 \varphi}$ is an integral constant.

The function $v$ is determined by use of Eq. (18) and (28)

$$
v=-\frac{a_{3}+b_{3}}{2 a_{2}} e^{-\left(a_{3}-b_{3}\right) t}
$$


and the second term in Eq.(30) leads to

$$
\frac{C C_{2} \int_{0}^{t} v d t}{x_{12}}=-\frac{C C_{2}}{a_{3}-b_{3}} e^{-\left(a_{3}-b_{3}\right) t}=B e^{-\left(a_{3}-b_{3}\right) t},
$$

where $B=-\frac{C C_{2}}{a_{3}-b_{3}}$ is an integral constant.

Since $C_{1}, C_{2}$ are integral constants, by choosing $C C_{1}=1$, and substituting expressions (31), (32) into Eq.(30) we can write the general solution of Eq.(26) as follows

$$
\begin{aligned}
\frac{1}{x}=-\frac{2 a_{2}}{a_{3}+b_{3}} & +A e^{\frac{a_{3}+b_{3}}{a_{1}+b_{1}} t}+B e^{-\left(a_{3}-b_{3}\right) t}= \\
& -\frac{2 a_{2}}{a_{3}+b_{3}}+e^{-\frac{a_{1} a_{3}-b_{1} b_{3}}{a_{1}+b_{1}} t}\left(A e^{\frac{b_{1} a_{3}-a_{1} b_{3}}{a_{1}+b_{1}} t}+B e^{-\frac{b_{1} a_{3}-a_{1} b_{3}}{a_{1}+b_{1}} t}\right),
\end{aligned}
$$

or in other form:

$$
\frac{1}{x}=-\frac{2 a_{2}}{a_{3}+b_{3}}+D e^{-\frac{a_{1} a_{3}-b_{1} b_{3}}{a_{1}+b_{1}} t} \operatorname{ch}\left[\frac{b_{1} a_{3}-a_{1} b_{3}}{a_{1}+b_{1}} t+\gamma\right],
$$

where $D, \gamma$ - integral constants.

By use of notation $\beta=\frac{a_{1} a_{3}-b_{1} b_{3}}{a_{1}+b_{1}}, m+\beta^{2}=-\frac{a_{3}^{2}-b_{3}^{2}}{a_{1}+b_{1}}$. Eq.(26) has the form

$$
\ddot{x}-2 \frac{\dot{x}^{2}}{x}+2 \beta \dot{x}+\frac{2 a_{2}\left(a_{3}-b_{3}\right)}{a_{1}+b_{1}} x^{2}-\left(1+\beta^{2}\right) x=0,
$$

general solution of which

$$
\begin{aligned}
& \frac{1}{x}=-\frac{2 a_{2}}{a_{3}+b_{3}}+D e^{-\beta t} \operatorname{ch}(t \sqrt{-m}+\gamma) \text { when } m<0, \\
& \frac{1}{x}=-\frac{2 a_{2}}{a_{3}+b_{3}}+D e^{-\beta t} \cos (t \sqrt{m}+\gamma) \text { when } m>0 .
\end{aligned}
$$

This solution was found in [4] by other method.

3.3. Case 3: $a_{1}-b_{1}=1$

In this case Eq.(1) has the form:

$$
\begin{aligned}
& \left(a_{1}+b_{1}\right) \ddot{x}+\left(a_{2}+b_{2}+2 a_{1} a_{2}-2 b_{1} b_{2}\right) x \dot{x} \\
& +\left(a_{2} d+b_{2} d+2 a_{1} a_{3}-2 b_{1} b_{3}\right) \dot{x}+\left(a_{2}^{2}-b_{2}^{2}\right) x^{3}+\left(a_{2}^{2} d-b_{2}^{2} d+2 a_{2} a_{3}-2 b_{2} b_{3}\right) x^{2} \\
& +\left(2 a_{2} a_{3} d-2 b_{2} b_{3} d+a_{3}^{2}-b_{3}^{2}+2 a_{2} b-2 b_{2} b\right) x+a_{3}^{2} d-b_{3}^{2} d+2 a_{3} b-2 b_{3} b=0 .
\end{aligned}
$$

According to (10) the constant particular solution to Eq. (37) is defined

$$
x_{1}+d=\frac{-\left(a_{3}-a_{2} d+b_{3}-b_{2} d\right)}{2\left(a_{2}+b_{2}\right)}+\frac{\sqrt{\delta}}{2\left(a_{2}+b_{2}\right)},
$$


where: $\delta=\left(a_{3}-a_{2} d+b_{3}-b_{2} d\right)^{2}-8 b\left(a_{2}+b_{2}\right)$.

Based on (18) function $v$ is calculated as:

$$
v=\frac{1}{x_{1}+d} e^{-\alpha t}
$$

where $\alpha=\left(a_{2}-b_{2}\right)\left(x_{1}+d\right)+a_{3}-a_{2} d-\left(b_{3}-b_{2} d\right)$.

Function $\mu$ can be determined by (20) and (39):

$$
\mu=\left(x_{1}+d\right) e^{-\beta t}\left[C_{1} e^{\alpha t}-\frac{C_{2}}{\left(x_{1}+d\right) \alpha}\right],
$$

where $\beta=a_{3}-a_{2} d-\left(b_{3}-b_{2} d\right)$.

According to Eq.(19) and using (40) the general solution to Eq.(37) can be obtained:

$$
x+d=\frac{\left(x_{1}+d\right)\left[A e^{\alpha t}-\frac{1}{\left(x_{1}+d\right) \alpha}\right]}{A e^{\alpha t}+B e^{\beta t}+\frac{\alpha-\beta}{\left(x_{1}+d\right) \alpha \beta}},
$$

where $A=\frac{C_{1}}{C_{2}}, B=\frac{C}{C_{2}}$ - integral constants.

Differentiating both sides of Eq. (41) with respect to $t$ yields

$$
\dot{x}=\frac{(\alpha-\beta)\left(x_{1}+d\right) A B e^{(\alpha+\beta) t}+\frac{\alpha}{\beta} A e^{\alpha t}+\frac{\beta}{\alpha} B e^{\beta t}}{\left[A e^{\alpha t}+B e^{\beta t}+\frac{\alpha-\beta}{\left(x_{1}+d\right) \alpha \beta}\right]^{2}} .
$$

Suppose initial conditions

$$
\left.x(t)\right|_{t=0}=x_{0},\left.\quad \dot{x}(t)\right|_{t=0}=\dot{x}_{0},
$$

are satisfied, then

$$
\begin{aligned}
& A=\frac{1}{\left(x_{1}+d\right) \alpha}-\frac{x_{0}+d}{(\alpha-\beta)\left(x_{1}-x_{0}\right)\left(x_{0}+d\right)-\dot{x}_{0}\left(x_{1}+d\right)}, \\
& B=\frac{1}{\left(x_{1}+d\right) \beta}-\frac{x_{1}-x_{0}}{(\alpha-\beta)\left(x_{1}-x_{0}\right)\left(x_{0}+d\right)-\dot{x}_{0}\left(x_{1}+d\right)}
\end{aligned}
$$

Now by use of mentioned above calculations we can seek general solutions of some non-linear equations, which usually have place in mechanical problems such as

$$
\ddot{x}+(2 \nu+3 \sigma x) \dot{x}+\lambda x^{3}+2 q x^{2}+k x+c=0 .
$$


Eq. (45) coincides entirely with Eq. (37) when putting

$$
\begin{aligned}
& a_{2}+b_{2}+2 a_{1} a_{2}-2 b_{1} b_{2}=3 \sigma\left(a_{1}+b_{1}\right), \\
& a_{2} d+b_{2} d+2 a_{1} a_{3}-2 b_{1} b_{3}=2 \nu\left(a_{1}+b_{1}\right), \\
& a_{2}^{2}-b_{2}^{2}=\lambda\left(a_{1}+b_{1}\right), \\
& a_{2}^{2} d-b_{2}^{2} d+2 a_{2} a_{3}-2 b_{2} b_{3}=2 q\left(a_{1}+b_{1}\right), \\
& 2 a_{2} a_{3} d-2 b_{2} b_{3} d+a_{3}^{2}-b_{3}^{2}+2 a_{2} b-2 b_{2} b=k\left(a_{1}+b_{1}\right), \\
& a_{3}^{2} d-b_{3}^{2} d+2 a_{3} b-2 b_{3} b=c\left(a_{1}+b_{1}\right) .
\end{aligned}
$$

The system of equations (46) consists of 6 equations with 6 unknowns $a_{2}, b_{2}, a_{3}, b_{3}$, $b, d$. So that 6 these unknowns can be determined through $a_{1}+b_{1}$ and 6 known parameters $\sigma, v, \lambda, q, k, c$, where $a_{1}+b_{1}$ can be chosen arbitrarily such that $a_{1}-b_{1}=1$. After that substituting just determined values into the expression of general solution (41) and integral constants (44) we can obtain the solution of Eq.(45) combined with initial conditions (43).

Note that Eq.(45) with all coefficients has a similar form of Van der Pol equation, and when parameter $\sigma=0$ has the form of Duffing equation, and when $\sigma=0, v=0$ - the form of $\mathrm{KdV}$ equation, respectively.

Explicit solution of the system of equations (46) can be received, it may be expressed as real or complex values. This problem requires further investigation.

\section{EXAMPLES}

4.1. Case: $a_{1}=1, b_{1}=0, b_{2}=0, b=0, d=0$

Eq (37) has the form

$$
\ddot{x}+\left(2 a_{3}+3 a_{2} x\right) \dot{x}+a_{2}^{2} x^{3}+2 a_{2} a_{3} x^{2}+\left(a_{3}^{2}-b_{3}^{2}\right) x=0 .
$$

Using calculations in Section 3.3 results

$$
x_{1}+d=-\frac{a_{3}+b_{3}}{a_{2}}, \quad \alpha=-2 b_{3}, \quad \beta=a_{3}-b_{3},
$$

and the general solution is defined

$$
\frac{1}{x}=\frac{a_{2}}{a_{3}^{2}-b_{3}^{2}}\left[-a_{3}+b_{3} \operatorname{th}\left(b_{3} t+\varphi\right)\right]+C_{0} \frac{e^{a_{3} t}}{\operatorname{ch}\left(b_{3} t+\varphi\right)},
$$

where $C_{0}, \varphi$ - integral constants.

Solution (48) was found in [5] by other method.

\subsection{Numerical case}

$a_{1}=1, b_{1}=0, b_{2}=0, b=0, d=0, a_{2}=0.2, a_{3}=-0.5, b_{3}=0.2$, with initial conditions: $x[0]=2, \dot{x}[0]=0.1$.

a. Seeking solution by numerical method

Numericalsolution $=N D$ Solve $\left[\left\{x^{\prime \prime}[t]+(2 a 3+3 a 2 * x[t]) * x^{\prime}[t]+a 2 \wedge 2 * x[t] \wedge 3+\right.\right.$ $\left.2 a 2 * a 3 * x[t] \wedge 2+(a 3 \wedge 2-b 3 \wedge 2) * x[t], x[0]=2, x^{\prime}[0]=0.1, x,\{t, 0,100\}\right]$. 
b. Seeking solution by analytical method

$$
\begin{aligned}
& \delta=\left(a_{3}-a_{2} d+b_{3}-b_{2} d\right)^{2}-8 b\left(a_{2}+b_{2}\right)=0.09 \\
& x_{1}+d=\frac{-\left(a_{3}-a_{2} d+b_{3}-b_{2} d\right)}{2\left(a_{2}+b_{2}\right)}+\frac{\sqrt{\delta}}{2\left(a_{2}+b_{2}\right)}=1.5 \\
& \alpha=\left(a_{2}-b_{2}\right)\left(x_{1}+d\right)+a_{3}-a_{2} d-\left(b_{3}-b_{2} d\right)=-0.4 \\
& \beta=a_{3}-a_{2} d-\left(b_{3}-b_{2} d\right)=-0.7 \\
& A=\frac{1}{\left(x_{1}+d\right) \alpha}-\frac{x_{0}+d}{(\alpha-\beta)\left(x_{1}-x_{0}\right)\left(x_{0}+d\right)-\dot{x}_{0}\left(x_{1}+d\right)}=2.77778 \\
& B=\frac{1}{\left(x_{1}+d\right) \beta}-\frac{x_{1}-x_{0}}{(\alpha-\beta)\left(x_{1}-x_{0}\right)\left(x_{0}+d\right)-\dot{x}_{0}\left(x_{1}+d\right)}=-0.15873 \\
& x+d=\frac{\left(x_{1}+d\right)\left[A e^{\alpha t}-\frac{1}{\left(x_{1}+d\right) \alpha}\right]}{A e^{\alpha t}+B e^{\beta t}+\frac{\alpha-\beta}{\left(x_{1}+d\right) \alpha \beta}}
\end{aligned}
$$

Plot $[\{x[t]$, Evaluate $[x[t] /$.numericalsolution $\},\{t, 0,100\}$, PlotStyle $\rightarrow\{$ Thick, Red, Blue $\}]$ (see Fig.1).

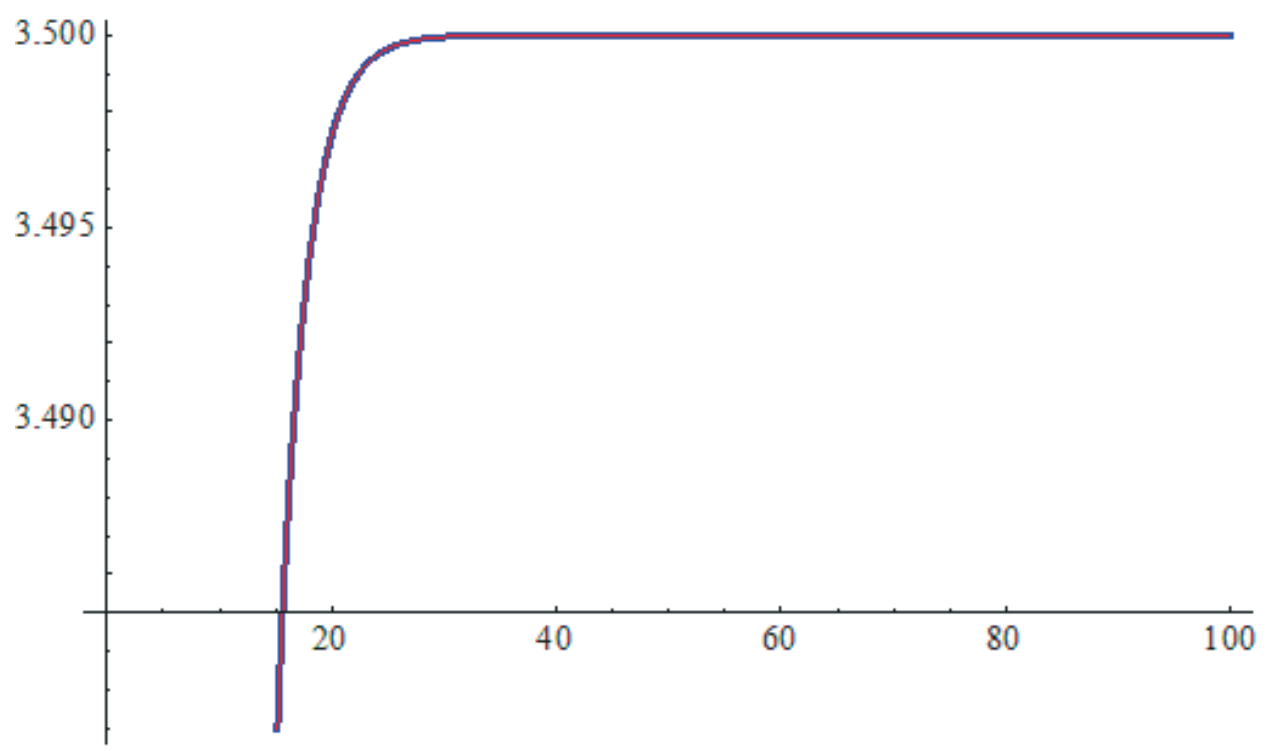

Fig. 1. Graph of exact solution $[\{x(t)$, Evaluate $[x[t] /$. numericalsolution $\},\{t, 0,100\}]$ to Eq. (47)

As can be observed, a very good agreement is obtained in this comparison study. 


\section{CONCLUSION}

- Equation of the form (1) is non-linear differential one, general exact solutions of which can be found. They include many particular cases, where exact solutions were defined by other methods.

- To obtain exact solutions of non-linear differential equations of the form (1) one needs to know important informations about particular solutions (8), (9), (10) and expressions (11), (12).

- Proposed method for finding general exact solution of non-linear differential equations of the form (1) is based upon a known particular solution and expressions (11), (12) in use of idea of the varying coefficient iteration method.

- Verification of the proposed method is proven by obtaining exact solutions in many particular cases. These solutions coincide with exact solutions obtained by other analytical methods and are in good agreement with results using numerical methods.

\section{REFERENCES}

[1] Nguyen Dang Bich, Ngo Dinh Bao Nam, Conditions for the approximated analytical solution of a parametric oscillation problem described by the Mathieu equation, Journal of Science, Mathematics - Physics, VNU, 21(2) (2006) 9 - 16.

[2] Dao Huy Bich, Plasticity theory and its applications, Publishing House of Civil Engineering, Hanoi, (2004).

[3] Nguyen Dang Bich, Nguyen Vo Thong, Aerodynamical instability of cylindrical bar supported on elastic hold with viscous damping, Journal of Mechanics, 16(3) (1994) 1 - 4.

[4] Dao Huy Bich, Nguyen Dang Bich, On the method for solving a class of non-linear differential equations in mechanics, Proceedings of the sixth National Congress on Mechanics, 3 - 5 December, Hanoi, 3 (1997) 11 - 17.

[5] Dao Huy Bich, Nguyen Dang Bich, Nguyen Vo Thong, The elastic aerodynamic problem with aerodynamical damping of turbulent characteristic, Proceedings of the ICCMS/IBST 2001, 28 - 29 March, Hanoi, Vietnam, 87 - 93. 